\title{
Differential search algorithm-based parametric optimization of electrochemical micromachining processes
}

\author{
Debkalpa Goswami and Shankar Chakraborty*
}

Department of Production Engineering, Jadavpur University, Kolkata - 700 032, West Bengal, India

\begin{tabular}{|c|c|}
\hline CHR ON I C L E & A B S T R A C T \\
\hline $\begin{array}{l}\text { Article history: } \\
\text { Received June } 22013 \\
\text { Received in revised format } \\
\text { August } 152013 \\
\text { Accepted August } 152013 \\
\text { Available online } \\
\text { August } 192013 \\
\text { Keywords: } \\
\text { Electrochemical micromachining } \\
\text { process } \\
\text { Differential search algorithm } \\
\text { Process parameter } \\
\text { Response }\end{array}$ & $\begin{array}{l}\text { Electrochemical micromachining (EMM) appears to be a very promising micromachining process } \\
\text { for having higher machining rate, better precision and control, reliability, flexibility, } \\
\text { environmental acceptability, and capability of machining a wide range of materials. It permits } \\
\text { machining of chemically resistant materials, like titanium, copper alloys, super alloys and } \\
\text { stainless steel to be used in biomedical, electronic, micro-electromechanical system and nano- } \\
\text { electromechanical system applications. Therefore, the optimal use of an EMM process for } \\
\text { achieving enhanced machining rate and improved profile accuracy demands selection of its } \\
\text { various machining parameters. Various optimization tools, primarily Derringer's desirability } \\
\text { function approach have been employed by the past researchers for deriving the best parametric } \\
\text { settings of EMM processes, which inherently lead to sub-optimal or near optimal solutions. In } \\
\text { this paper, an attempt is made to apply an almost new optimization tool, i.e. differential search } \\
\text { algorithm (DSA) for parametric optimization of three EMM processes. A comparative study of } \\
\text { optimization performance between DSA, genetic algorithm and desirability function approach } \\
\text { proves the wide acceptability of DSA as a global optimization tool. }\end{array}$ \\
\hline
\end{tabular}

\section{Introduction}

Micromachining refers to those processes where material removal takes place in small dimensions ranging from $1-999 \mu \mathrm{m}$. It also implies machining which cannot be achieved directly using the conventional processes. Recent requirements of the advanced manufacturing industries have forced the introduction of micro- and nano-components into various products, ranging from biomedical applications to chemical micro-reactors and sensors. Micromachining technology plays an important role in miniaturization of the components. It helps in machining of micro-slots, complex surfaces and holes, which require to be produced in large numbers in a single workpiece, especially in electronic, space and automobile industries. If those micro-components are machined using the conventional machining processes, there may be occasional problems of tool wear, tool rigidity, heat generation at

* Corresponding author. Tel: +91-33-2414-6153

E-mail: s_chakraborty00@yahoo.co.in (S. Chakraborty)

(C) 2014 Growing Science Ltd. All rights reserved.

doi: $10.5267 / j$. .ijiec.2013.08.003 
the tool-workpiece interface etc. Sometimes, it is also difficult to machine complex and intricate shapes employing the conventional processes. Since there will be continuous demand for miniaturization for efficient space utilization with better quality of products, micromachining technology will become still more important in the future (Bhattacharyya et al., 2004).

When electrochemical machining (ECM) process is used in the micron range of application, it is called electrochemical micromachining (EMM) process. EMM is a very promising micromachining technology due to its various inherent advantages, like high material removal rate (MRR), better precision and control, reliability, flexibility, environmental acceptability and green manufacturing. It is widely used to machine hard materials at a high MRR, without affecting the tensile strength of the workpiece material and its other physical properties, while ensuring a low surface roughness. It also permits machining of chemically resistant materials like, titanium, copper alloys, super alloys and stainless steel, which are now widely used in biomedical, electronic, micro-electromechanical system (MEMS) and nano-electromechanical system (NEMS) applications. EMM process could be used as one the best micromachining techniques for machining electrically conducting, tough and difficult to machine materials with appropriate combination of machining parameters. The desired quality of the workpiece in EMM process can only be generated through combinational control of its various process parameters. Therefore, the optimal use of EMM process for achieving higher MRR, improved profile accuracy and surface quality demands proper control of its process parameters. However, to exploit the full potential of EMM process, research is still needed to improve the machining accuracy by controlling its different machining parameters and determining the optimal parametric combination. An excellent review on the machining mechanism of EMM process can be found in Bhattacharyya et al. (2004) and Cao et al. (2012).

\section{Survey of past research}

Bhattacharyya et al. (2001) designed and developed an EMM setup comprising of various mechanical machining components, electrical system, an electrolyte flow system and a microprocessor-controlled end-gap controlling system. Bhattacharyya et al. (2002) developed an EMM system setup for performing basic and fundamental research in the area of EMM, fulfilling the objectives of micromachining. Bhattacharyya and Munda $\left(2003^{\mathrm{a}}\right)$ investigated the influence of machining voltage, electrolyte concentration, pulse-on time and frequency of pulsed power supply on MRR and accuracy for effective utilization of an ECM system for micromachining operation. Bhattacharyya and Munda $\left(2003^{\mathrm{b}}\right)$ developed an EMM setup to meet the micromachining requirements, and indicated the most effective zone of predominant process parameters, such as machining voltage and electrolyte concentration for providing the appreciable amount of MRR with less overcut. Bhattacharyya et al. (2005) studied the influence of various EMM process parameters, like machining voltage, electrolyte concentration, pulse period and frequency on MRR, accuracy and surface finish in microscopic domain. Kurita et al. (2006) determined the optimal values of machining voltage, machining pulse length, amplitude of the electrode for flushing out contamination and electrolyte concentration, and carried out three-dimensional shape micromachining at the derived optimal conditions. Bhattacharyya et al. (2007) carried out experiments to determine the optimal values of different EMM process parameters, such as micro-tool vibration frequency, amplitude and electrolyte concentration for producing micro-holes with higher accuracy and appreciable amount of MRR. Using response surface methodology (RSM), Munda et al. (2007) correlated the interactive and higher-order influences of various machining parameters on the combined effect of micro-spark and stray-current machining in an EMM process. Munda and Bhattacharyya (2008) developed a mathematical model for correlating the interactive and higher-order influences of various EMM process parameters, i.e. machining voltage pulse on/off ratio, machining voltage, electrolyte concentration, voltage frequency and tool vibration frequency on MRR and radial overcut (ROC). Munda et al. (2010) developed mathematical models for depicting the interactive and higher-order influences of various machining parameters on ROC in an EMM process. The developed mathematical models would be useful to find out the optimal parametric 
setting to produce better quality machined products at a higher rate. Li and Niu (2010) carried out experiments to investigate the influence of some EMM process parameters, such as pulse frequency, feed rate of tool, machining voltage and ultrasonic frequency on the machining accuracy of microholes. Malapati and Bhattacharyya (2011) considered five EMM process parameters, i.e. pulse frequency, machining voltage, duty ratio, electrolyte concentration and micro-tool feed rate, and studied their influences on two important responses, i.e. MRR and machining accuracy, during microchannel generation. The optimal values of those process parameters were also found out employing desirability function approach. Malapati et al. (2011) studied the effect of various EMM process parameters, i.e. machining voltage, electrolyte concentration, frequency pulse period and duty factor on MRR and machining accuracy to meet the micromachining requirements. Malapati et al. (2012) investigated the influence of different process parameters on micromachining criterion using Taguchi method of robust design, and developed second order regression models to search for the best parametric combination to achieve the desired micromachining characteristics. Mithu et al. (2012) fabricated micro nozzles and micro pockets by EMM process. Rao and Patel (2012) introduced the elitism concept in teaching-learning-based optimization algorithm and investigated the effects of common controlling parameters, like population size and number of generations on the performance of that algorithm. Its optimization performance was also compared with that of other well known evolutionary algorithms. Thanigaivelan and Arunachalam (2013) optimized the process parameters for EMM operation of 304 stainless steel using grey relational analysis. Machining voltage, pulse on-time, electrolyte concentration and tool tip shapes were selected as the typical process parameters, and machining rate and overcut were the responses.

The past researchers have already applied various optimization tools and techniques, mainly Taguchi method and Derringer's desirability function approach for finding out the optimal settings of EMM process parameters. But, those adopted approaches have several inherent limitations (especially generation of near optimal or sub-optimal solutions and high computational time) which compel the authors to explore the feasibility and acceptability of an almost unexplored optimization tool, i.e. differential search algorithm (DSA) for parametric optimization of EMM processes.

\section{Differential search algorithm}

The sustainability and efficiency of food sources available in nature (pastures, water supplies etc.) typically vary with the periodicity of seasonal changes. Thus, it is the characteristic of many species (eusocial, subsocial or presocial) to show seasonal migratory behavior to move to a more thriving habitat (Krebs and Davies, 1997). The migrating species of living beings constitute a superorganism, containing a large number of individuals.

There are a number of stochastic computational intelligence algorithms that model the behavior of superorganisms. Some of the most popular algorithms in this category include particle swarm optimization (PSO), cuckoo search (CS), artificial bee colony (ABC) algorithm, ant colony optimization (ACO) etc. The differential search algorithm (DSA) is the latest addition to this group.

Movement of a superorganism can be described by a Brownian-like random walk model (Trianni et al., 2011). In DSA, it is assumed that a population made up of random solutions of the respective problem corresponds to an artificial superorganism migration (Civicioglu, 2012). This superorganism tends to migrate to a global minimum of the problem. In the course of this migration, the artificial superorganism tests whether some randomly selected positions are temporarily suitable for stop-over. If it is so, members of the superorganism that made this discovery immediately settle at this discovered position and continue their migration from here on.

A detailed pseudo code for DSA (Civicioglu, 2012) is presented below, and its nuances are explained in the subsequent paragraphs of this section. 


\section{Pseudo code: Differential search algorithm}

Require:

$N$ : Size of the population, where $i=\{1,2,3, \ldots, N\}$

$D$ : Dimension of the problem

$G$ : Number of maximum generation

1: $\quad$ Superorganism $=$ initialize (), where Superorganism $=\left[\right.$ ArtificialOrganism $\left._{i}\right]$

2: $\quad y_{i}=$ Evaluate(ArtificialOrganism $_{i}$ )

3: $\quad$ for cycle $=1: G$ do

4: $\quad$ donor $=$ Superorganism Random_Shuffling $(i)$

5: $\quad$ Scale $=\operatorname{randg}\left[2 \cdot \operatorname{rand}_{1}\right] \cdot\left(\right.$ rand $_{2}-$ rand $\left._{3}\right)$

6: $\quad$ StopoverSite $=$ Superorganism + Scale $\cdot($ donor - Superorganism $)$

7: $\quad p_{1}=0.3 \cdot$ rand $_{4}$ and $p_{2}=0.3 \cdot$ rand $_{5}$

8: $\quad$ if rand $_{6}<$ rand $_{7}$ then

9: $\quad$ if rand $_{8}<p_{1}$ then

10: $\quad r=\operatorname{rand}(N, D)$

11:

12:

13:

14:

15:

$16:$

17:

18:

19:

20:

21:

22:

23:

24:

25:

26:

27:

28:

29:

for Counter $1=1: N$ do $r($ Counter $1,:)=r\left({\text { Counter } 1,:)<\text { rand }_{9}}\right.$

end for

else

$r=\operatorname{ones}(N, D)$

for Counter2 $=1: N$ do

$r($ Counter $2, \operatorname{randi}(D))=r($ Counter $2, \operatorname{randi}(D))<\operatorname{rand}_{10}$ end for

\section{end if}

else

$$
r=\operatorname{ones}(N, D)
$$

for Counter3 $=1: N$ do

$$
d=\operatorname{randi}\left(D, 1,\left[p_{2} \cdot \text { rand } \cdot D\right\rceil\right)
$$

for Counter4 $=1$ : size $(d)$ do

$$
r(\text { Counter } 3, d(\text { Counter } 4))=0
$$

\section{end for}

\section{end for}

\section{end if}

individuals $_{I, J} \leftarrow r_{I, J}>0 \mid I \in i, J \in[1, D]$

30: $\quad$ StopoverSite(individual $s_{I, J}$ ) $:=$ Superorganism(individual $s_{I, J}$ )

31: if StopoverSite ${ }_{i, j}<$ low $_{i, j}$ or StopverSite ${ }_{i, j}>$ up $_{i, j}$ then

32:

33:

$$
\text { StopoverSite }_{i, j}:=\text { rand } \cdot\left(\text { up }_{j}-\text { low }_{j}\right)+\text { low }_{j}
$$

34:

end if

$y_{\text {Stopoversite } ;}=$ evaluate $\left(\right.$ StopoverSite $\left.{ }_{i}\right)$

35:

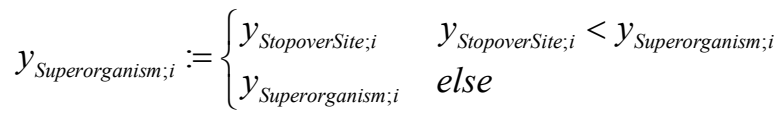

36:

ArtificialOrganism $_{i}:= \begin{cases}\text { StopoverSite }_{i} & y_{\text {StopoverSite } ;}<y_{\text {Superorganism } ; i_{i}} \\ \text { ArtificialOrganism }_{i} & \text { else }\end{cases}$

37: end for

A member of an artificial organism, in its initial position, is defined by the following equation: 
$x_{i, j}=$ rand $\cdot\left(u_{j}-\right.$ low $\left._{j}\right)+$ low $_{j}$

Subsequently, the artificial organisms are defined by $X_{i}=\left[x_{i, j}\right]$ and the superorganism made up of these artificial organisms is denoted by Superorganism ${ }_{g}=\left[X_{i}\right]$. The mechanism of finding a stop-over site involves implementation of the Brownian-like random walk model (Trianni et al., 2011). The randomly selected individuals of the artificial organisms move towards the targets of donor $=\left[X_{\text {Random_Shuffling(i })}\right]$ in order to discover these stop-over sites, which are pivotal for a successful migration. The magnitude of the change in positions of the members of the artificial organisms is governed by the Scale factor. The value of Scale is determined using a gamma random number generator (randg), which in turn, is controlled by a uniform random number generator ( rand). This coding structure (refer to line 5 of pseudo code) allows the artificial superorganism to radically change direction within the habitat.

The expression for a stop-over site position is given by the following equation:

$$
\text { StopoverSite }=\text { Superorganism }+ \text { Scale } \times(\text { donor }- \text { Superorganism })
$$

The members which participate in the search process for stop-over sites are determined by a stochastic scheme (lines 8-28 of pseudo code). Since this process is completely random, there is a probability that an element of the stop-over site is beyond the limits of the habitat (i.e. search space). To make the code foolproof against this situation, a suitable check is hence devised (lines 31-33 of pseudo-code).

If a stop-over site is more thriving than the sources owned by the artificial organism, the artificial organism moves to the newly discovered stop-over site. While the artificial organisms change site, the superorganism continues its migration towards the global optimum.

Two unique characteristics of DSA make it a successful search tool for solution of multi-modal functions. Firstly, DSA may simultaneously use more than one individual; and secondly, this algorithm has no inclination to go towards the so-called best possible solution of the problem. These features are unlike the other existing algorithms, like PSO, ABC etc. These traits are also evident from the nature of the scatter plots generated by DSA (given in sub-section 4.1 of this paper). The scatter diagrams generated by algorithms, like ABC and PSO clearly show a clustering of navigated data points near the optima (Samanta \& Chakraborty, 2011). But in case of DSA, such a behavior is not to be observed (refer to scatter diagrams given in sub-section 4.1 of this paper).

The DSA has only two control parameters, $p_{1}$ and $p_{2}$ (line 7 of pseudo code). Civicioglu (2012) conducted detailed tests to determine the most appropriate values for these two control parameters, and concluded that this algorithm is not much sensitive to the values of $p_{1}$ and $p_{2}$. The straightforward algorithmic structure of DSA allows for its easy application in practical engineering problems of interest.

\section{Illustrative examples}

In order to prove and validate the usefulness, applicability and solution accuracy of DSA in solving complex polynomial mathematical functions, the following three real time optimization problems are considered. In all these three cases, the past researchers developed RSM-based equations correlating the responses with various EMM process parameters and adopted different optimization tools to search out the optimal combinations of the EMM process parameters for enhanced machining performance. In this paper, those RSM-based equations are considered for both single and multi-objective optimization of the responses employing DSA tool. 


\subsection{Example 1}

Malapati and Bhattacharyya (2011) developed an EMM setup to study the influence of various process parameters, like pulse frequency, machining voltage, duty ratio, electrolyte concentration and microtool feed rate on MRR, width-overcut (WOC), length-overcut (LOC) and linearity during microchannel generation. In order to develop the relationships between the considered five EMM process parameters and responses, 32 experiments were conducted on bare copper plates $(15 \mathrm{~mm} \times 10 \mathrm{~mm} \times 0.15 \mathrm{~mm})$ using a central composite half factorial second-order rotatable design plan. Each of the EMM process parameters was set at five different levels, as given in Table 1. Among the considered responses, MRR is the ratio of difference in weights (final weight - initial weight) to the machining time, WOC is the excess dimensional machining across the micro-channel, LOC is the excess dimensional machining along the micro-channel and linearity is defined as the uniformity of width along the length of the generated micro-channel. Using the experimental data and with the help of MINITAB software, Malapati and Bhattacharyya (2011) developed four RSM-based equations for the considered responses, as given by Eqs. (3-6), to explore the interactive and higher-order effects of various EMM process parameters on the responses.

\section{Table 1}

EMM process parameters along with their levels (Malapati and Bhattacharyya, 2011)

\begin{tabular}{lcccccc}
\hline Parameter & Unit & \multicolumn{4}{c}{ Levels } \\
\cline { 2 - 6 } & & -2 & -1 & 0 & +1 \\
\hline Pulse frequency $\left(x_{1}\right)$ & $\mathrm{kHz}$ & 40 & 50 & 60 & 70 & 80 \\
Machining voltage $\left(x_{2}\right)$ & $\mathrm{V}$ & 7 & 8 & 9 & 10 & 11 \\
Duty ratio $\left(x_{3}\right)$ & $\%$ & 30 & 40 & 50 & 60 & 70 \\
Electrolyte concentration $\left(x_{4}\right)$ & $\mathrm{g} / \mathrm{l}$ & 50 & 60 & 70 & 80 & 90 \\
Micro-tool feed rate $\left(x_{5}\right)$ & $\mu \mathrm{m} / \mathrm{sec}$ & 150 & 175 & 200 & 225 \\
\hline
\end{tabular}

$$
\begin{aligned}
& Y_{M R R}=2.59446+0.237004 x_{2}+0.426029 x_{3}+0.340938 x_{4}-0.385790 x_{1}{ }^{2}+0.295540 x_{2}{ }^{2}- \\
& 0.299427 x_{3}^{2}+0.224331 x_{1} x_{5}+0.327156 x_{2} x_{3}-0.243256 x_{2} x_{4} \\
& Y_{W O C}=0.124856+0.0288208 x_{4}+0.0270208 x_{5}-0.0173807 x_{3}^{2}+0.0140443 x_{5}^{2}+ \\
& 0.0203563 x_{1} x_{4}-0.0257063 x_{1} x_{5}-0.0209313 x_{2} x_{3} \\
& Y_{L O C}=0.403467+0.0526542 x_{2}+0.0604563 x_{1} x_{2}+0.0457563 x_{1} x_{3}+0.0344187 x_{1} x_{4}- \\
& 0.166544 x_{2} x_{4}-0.0554562 x_{2} x_{5}+0.0495813 x_{3} x_{4}+0.0413687 x_{3} x_{5}+0.0534565 x_{4} x_{5} \\
& Y_{\text {Linearity }}=0.0721852+0.00868750 x_{1}+0.0112125 x_{2}+0.0171458 x_{4}-0.0128688 x_{1} x_{3}+ \\
& 0.0151188 x_{1} x_{4}+0.00841875 x_{2} x_{4}-0.0116062 x_{2} x_{5}-0.00749375 x_{4} x_{5}
\end{aligned}
$$

\section{Single objective optimization}

In single objective optimization, all the four responses of the considered EMM process, i.e. MRR, WOC, LOC and linearity, are separately optimized using DSA. Among these responses, MRR needs to be maximized and the remaining three responses are to be minimized. For all these single objective optimization problems, the constraints are set as $40 \leq x_{1} \leq 80,7 \leq x_{2} \leq 11,30 \leq x_{3} \leq 70,50 \leq x_{4} \leq 90$ and $150 \leq x_{5} \leq 250$. In Table 2 , the results of single objective optimization of the responses are presented and it is observed that different combinations of the optimal EMM process parameters are attained by DSA for different responses. It may simply become impossible for the process engineers to set different EMM process parameter settings for attaining the desired values of the responses. This compels implementation of multi-objective optimization technique for achieving a unique combination of process parameters to simultaneously attain all the preferred values of the responses. Table 2 also compares the optimization performance of DSA with that of genetic algorithm (GA) which has already become a quite prominent population-based approach for dealing with complex optimization problems. It is quite evident from the optimization results of Table 2 that DSA outperforms GA with respect to 
the values of all the considered responses. While solving the above-mentioned single objective optimization problems on an Intel® Core $^{\mathrm{TM}}$ i5-2450M CPU @ 2.50 GHz with 4.00 GB RAM computing platform, the average computational time taken by DSA is $8.11 \mathrm{sec}$, whereas, GA takes an average of $12.41 \mathrm{sec}$ to solve those problems. Fig. 1 compares the convergence of DSA and GA for all the four responses and it is clear that DSA is quite faster than GA in achieving the optimal values of the responses. As Malapati and Bhattacharyya (2011) did not perform any single objective optimization of the responses, the results of DSA cannot be compared with theirs.

Table 2

Single objective optimization results for Example 1

\begin{tabular}{ccccccccc}
\hline Method & Response & Objective & Value & $x_{1}$ & $x_{2}$ & $x_{3}$ & $x_{4}$ & $x_{5}$ \\
\hline \multirow{5}{*}{ DSA } & MRR & Maximize & 5.6223 & 66.3833 & 7.0000 & 30.0000 & 50.0000 & 159.6055 \\
& WOC & Minimize & $7.05 \mathrm{E}-07$ & 61.0216 & 11.0000 & 45.6017 & 54.9639 & 172.8989 \\
& LOC & Minimize & $2.68 \mathrm{E}-05$ & 67.7531 & 9.3063 & 70.0000 & 84.1044 & 201.5386 \\
& Linearity & Minimize & $3.19 \mathrm{E}-06$ & 79.1782 & 7.0000 & 68.3325 & 64.1964 & 150.0000 \\
\hline \multirow{3}{*}{ GA } & MRR & Maximize & 3.9635 & 60.6760 & 7.0000 & 50.0200 & 77.8400 & 224.1500 \\
& WOC & Minimize & $6.85 \mathrm{E}-05$ & 77.7851 & 10.4260 & 62.0426 & 87.4213 & 250.0000 \\
& LOC & Minimize & $5.19 \mathrm{E}-04$ & 60.8481 & 7.4079 & 30.0000 & 67.2276 & 250.0000 \\
& Linearity & Minimize & $7.14 \mathrm{E}-04$ & 72.5229 & 7.5649 & 34.6474 & 87.4393 & 229.2281 \\
\hline
\end{tabular}
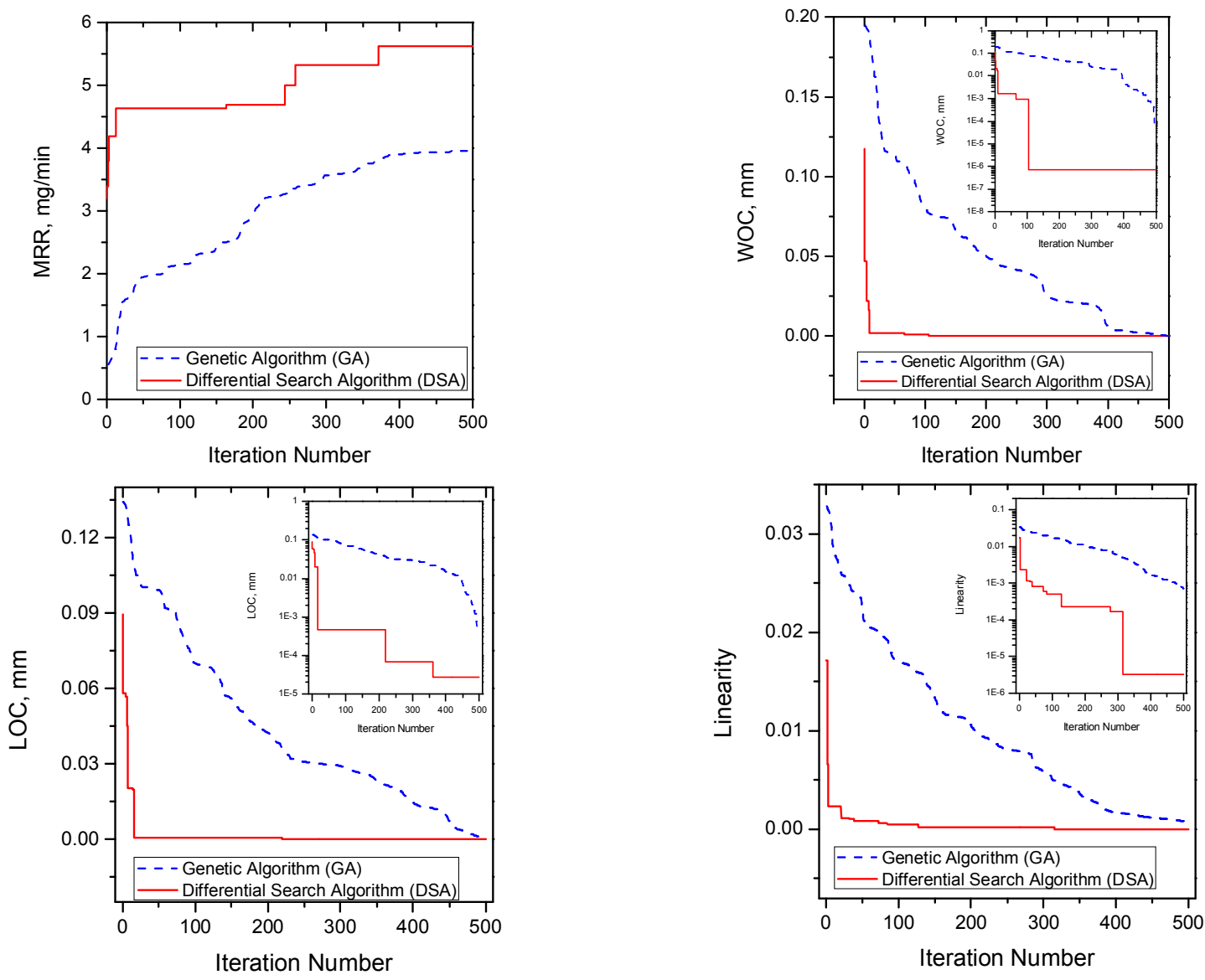

*Inset plot: vertical axis in logarithmic scale

Fig. 1. Convergence diagrams of DSA and GA for Example 1

Malapati and Bhattacharyya (2011) observed that MRR would increase with the increasing values of duty ratio up to a level of duty ratio $=50 \%$, and then it would start decreasing rapidly. Due to increase in duty ratio, the on-time of pulse period would increase, causing an increase in MRR. Usually, the dissolved machining products are flushed out from the machining zone during off-time of pulse period. 
Increase in duty ratio would also increase the dissolution rate, and these products cannot be efficiently flushed out, once the duty ratio crosses the limit of $50 \%$. Up to $50 \%$ duty ratio, the on-time is lower than off-time of pulse period, hence, the dissolved products can be flushed out easily. Beyond 50\% duty ratio, the dissolved products may pile up in the machining zone, disrupting the dissolution process and reducing the MRR. It was also found out that MRR would increase with electrolyte concentration. At higher concentration, more ions would be associated with machining, thus increasing the current density that would lead to higher MRR. Due to increase in machining voltage, machining current would also increase, leading to increase in current density which causes higher MRR. It was also observed that the value of MRR would increase non-linearly with increasing values of machining voltage. At higher machining voltage, ions associated with the machining process would be more, which may have an impact in enhancing the value of MRR.
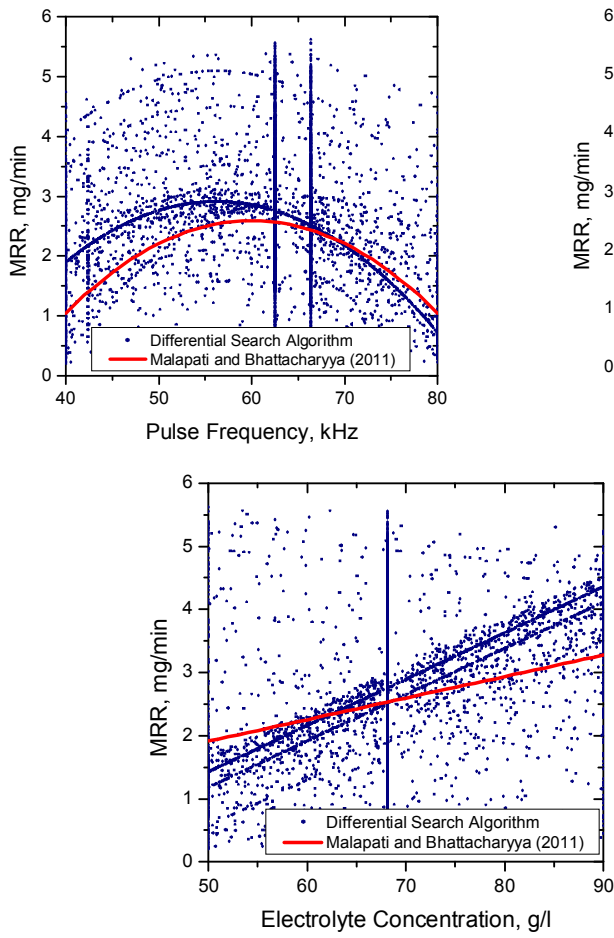
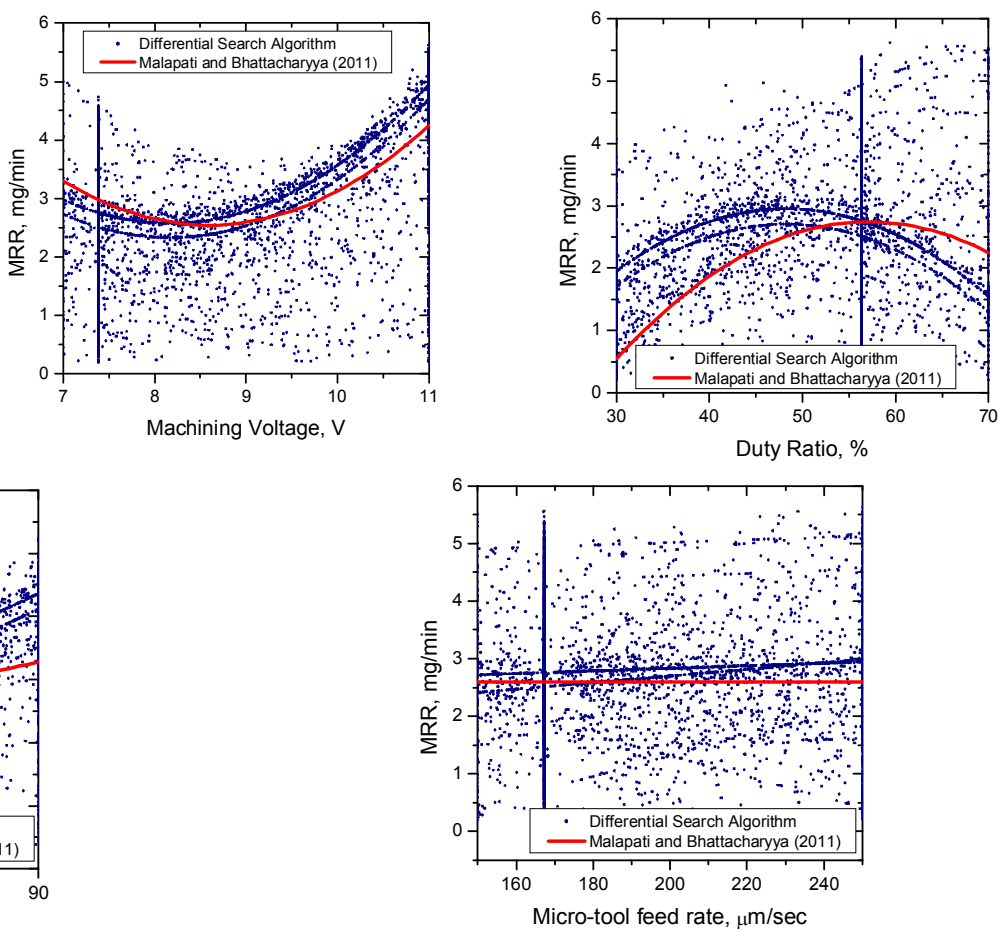

Fig. 2. Change of MRR with respect to various EMM process parameters

It was observed that WOC would be the minimum for lower duty ratio and machining voltage. Increase in duty ratio would increase the dissolution rate and, at the same time, decrease the off-time. So these dissolved products cannot be flushed out completely from the machining zone during a short span of off-time; they disturb the dissolution process and sometimes stick between the micro-tool and the workpiece, causing poor localization effects which in turn cause larger overcut. Furthermore, at higher machining voltage, electrochemical reactions generate hydrogen gas bubbles, which break down resulting in the occurrence of micro-sparks, causing uncontrollable material removal in the periphery of the micro-channel, which, in turn, produces larger overcut. Figures 2 and 3 respectively exhibit the variations of MRR and WOC with varying values of the EMM process parameters. These scatter diagrams clearly corroborate with the findings of Malapati and Bhattacharyya (2011). Similar types of scatter diagrams exhibiting variations of LOC and linearity are also developed which are not shown here due to lack of space. 

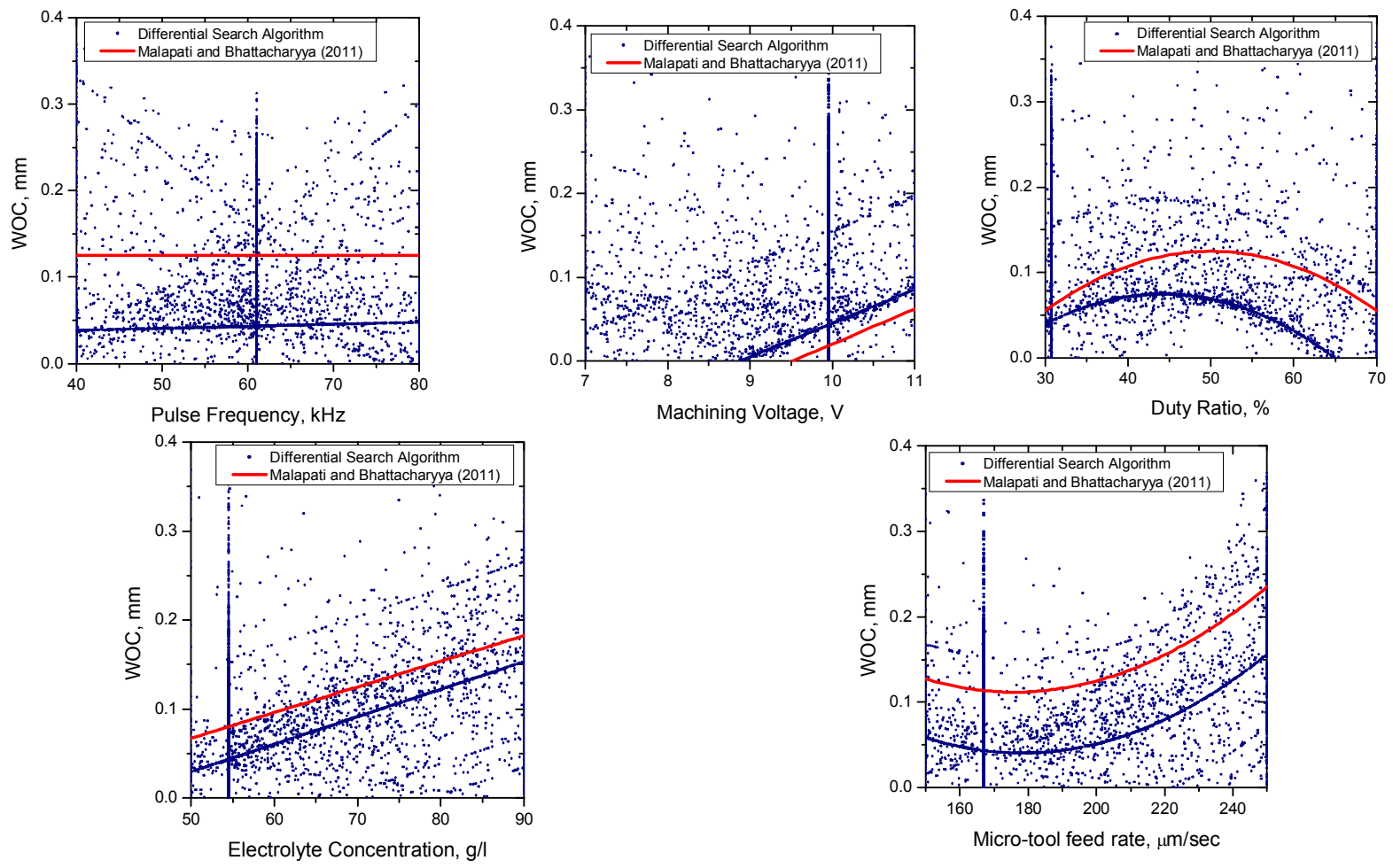

Fig. 3. Variations of WOC for different EMM process parameters

\section{Multi-objective optimization}

In multi-objective optimization, all the four responses are simultaneously optimized and for this, the following combined objective function is developed.

Minimize $\mathrm{Z}_{1}=\frac{w_{1} \times Y_{W O C}}{(W O C)_{\min }}+\frac{w_{2} \times Y_{L O C}}{(L O C)_{\min }}+\frac{w_{3} \times Y_{\text {Linearity }}}{(\text { Linearity })_{\min }}-\frac{w_{4} \times Y_{M R R}}{(M R R)_{\max }}$,

where $Y_{W O C}, Y_{L O C}, Y_{\text {Linearity }}$ and $Y_{M R R}$ are the RSM-based equations for WOC, LOC, Linearity and MRR respectively as developed using the experimental data; $w_{1}, w_{2}, w_{3}$ and $w_{4}$ are the weight/priorities assigned to WOC, LOC, Linearity and MRR respectively; and (WOC $)_{\min },(L O C)_{\min }$, (Linearity) $\min$ and $(M R R)_{\max }$ are respectively the optimal values of the considered responses as obtained from the single objective optimization results. The weights need to be assigned to the responses in such a way that they must add up to one. Sometimes, analytic hierarchy process may be helpful to determine the weight values to be allocated to the responses. In this case, equal weights, i.e. $w_{1}=w_{2}=w_{3}=w_{4}=0.25$, are assigned to all the four responses. The same set of constraints as considered for single objective optimization is also used here for multi-objective optimization.

After solving the combined objective function of Eq. (7) using DSA, the multi-objective optimization results of Table 3 are obtained. It is observed that a combination of EMM process parameters given by pulse frequency $=80.0000 \mathrm{kHz}$, machining voltage $=8.3340 \mathrm{~V}$, duty ratio $=64.6364 \%$, electrolyte concentration $=69.1625 \mathrm{~g} / 1$ and micro-tool feed rate $=181.4039 \mu \mathrm{m} / \mathrm{sec}$ provides the simultaneously optimized values of the four responses as MRR $=4.8279 \mathrm{mg} / \mathrm{min}, \mathrm{WOC}=0.00006 \mathrm{~mm}, \mathrm{LOC}=0.0861$ $\mathrm{mm}$ and linearity $=0.00053$. The minimum objective function value $\left(Z_{1}\right)$ is achieved as -0.0341 . After solving the multi-objective optimization problem for the EMM process using composite desirability function approach, Malapati and Bhattacharyya (2011) determined the optimal combination of various machining parameters as pulse frequency of $52.2818 \mathrm{kHz}$, machining voltage of $10.1033 \mathrm{~V}$, duty ratio of $68.3890 \%$, electrolyte concentration of $85.1515 \mathrm{~g} / \mathrm{l}$ and micro-tool feed rate of $208.5860 \mu \mathrm{m} / \mathrm{sec}$, 
where maximum MRR (3.1039 $\mathrm{mg} / \mathrm{min}$ ) and minimum values of accuracy (WOC of $0.0003 \mathrm{~mm}$, LOC of $0.1676 \mathrm{~mm}$ and linearity of 0.0691 ) were achieved. It can be concluded that the optimal values of all the four responses as derived by DSA are far better than those achieved by Malapati and Bhattacharyya (2011) through their experiments.

Table 3

Multi-objective optimization results for Example 1

\begin{tabular}{ccccccccc}
\hline$x_{1}$ & $x_{2}$ & $x_{3}$ & $x_{4}$ & $x_{5}$ & WOC & LOC & Lin & MRR \\
\hline 80.0000 & 8.3340 & 64.6364 & 69.1625 & 181.4039 & $6.03 \mathrm{E}-05$ & 0.0861 & $5.33 \mathrm{E}-04$ & 4.8279 \\
\hline
\end{tabular}

\subsection{Example 2}

Munda and Bhattacharyya (2008) considered pulse on/off ratio, machining voltage, electrolyte concentration, voltage frequency and tool vibration frequency during EMM operation to investigate their effects on MRR and radial overcut (ROC) of the machined components. The experimentation plan was designed not only to obtain the optimal scheme for multi-variable experimentation, but also to perform studies for exploring the interactive and higher-order effects of various process parameters. A stainless steel wire of diameter $335 \mu \mathrm{m}$ was used as the micro tool for the experimentation and the workpiece specimens were $15 \times 10 \times 0.15 \mathrm{~mm}$ bare copper plates. A central composite half fraction second-order rotatable design with 32 experiments was adopted for studying the relationship between the process parameters and responses. The original values of EMM process parameters with their corresponding levels are shown in Table 4. The developed RSM-based equations for MRR and ROC are respectively given in Eqs. (5-6).

\section{Table 4}

EMM process parameters and their levels (Munda and Bhattacharyya, 2008)

\begin{tabular}{lcccccc}
\multicolumn{1}{c}{ Parameter } & Unit & \multicolumn{4}{c}{ Levels } & \\
& & -2 & -1 & 0 & +1 \\
\hline Pulse on/off ratio $\left(x_{1}\right)$ & & 0.5 & 1.0 & 1.5 & 2.0 & 2.5 \\
Machining voltage $\left(x_{2}\right)$ & $\mathrm{V}$ & 2.5 & 3.0 & 3.5 & 4.0 & 4.5 \\
Electrolyte concentration $\left(x_{3}\right)$ & $\mathrm{g} / \mathrm{l}$ & 10 & 15 & 20 & 25 & 30 \\
Voltage frequency $\left(x_{4}\right)$ & $\mathrm{Hz}$ & 35 & 40 & 45 & 50 & 55 \\
Tool vibration frequency $\left(x_{5}\right)$ & $\mathrm{Hz}$ & 100 & 150 & 200 & 250 & 300 \\
\hline
\end{tabular}

$Y_{M R R}=-1.78917+0.111858 x_{1}+1.36263 x_{2}-0.0864044 x_{3}+0.0231122 x_{4}-0.00139639 x_{5}-$ $0.201666 x_{1}^{2}-0.0860582 x_{2}^{2}-0.000145752 x_{3}^{2}-0.000319532 x_{4}^{2}+3.893684 \times 10^{-6} x_{5}^{2}-$ $0.0704326 x_{1} x_{2}+0.00838936 x_{1} x_{3}+0.00275664 x_{1} x_{4}+0.00178484 x_{1} x_{5}+0.00870264 x_{2} x_{3}-$

$0.00700764 x_{2} x_{4}-0.00105004 x_{2} x_{5}+0.00125437 x_{3} x_{4}+0.0000247626 x_{3} x_{5}+0.0000181174 x_{4} x_{5}$

$Y_{R O C}=-1.08149+1.21039 x_{1}+0.448639 x_{2}-0.0821333 x_{3}+0.0247783 x_{4}-0.00258589 x_{5}+$ $0.0198541 x_{1}^{2}+0.0554876 x_{2}^{2}+0.00108447 x_{3}^{2}+0.000640329 x_{4}^{2}+2.817205 \times 10^{-6} x_{5}^{2}-$ $0.139966 x_{1} x_{2}-0.00133867 x_{1} x_{3}-0.0161759 x_{1} x_{4}-5.41335 \times 10^{-5} x_{1} x_{5}-0: 00307591 x_{2} x_{3}-$ $0.0163201 x_{2} x_{4}+0: 000831331 x_{2} x_{5}+0.000786541 x_{3} x_{4}+0.0000725981 x_{3} x_{5}$

$-6.94181 \times 10^{-5} x_{4} x_{5}$

\section{Single objective optimization}

Now employing DSA, the RSM-based equations, as given in Eqns. (8)-(9) for the two responses, i.e. MRR and ROC, are separately optimized. In this case, MRR needs to be maximized while minimization of ROC is required. For optimizing the RSM-based equations for the two responses, the constraints are set as $0.5 \leq x_{1} \leq 2.5,2.5 \leq x_{2} \leq 4.5,10 \leq x_{3} \leq 30,35 \leq x_{4} \leq 55$ and $100 \leq x_{5} \leq 300$. The results of single objective optimization using DSA are provided in Table 5. Again, two different sets of optimal EMM process parameter combinations are derived for the two separate responses. Table 5 also 
compares the optimal values of the responses as derived by DSA with those obtained by GA. It is again proved that DSA is superior to GA with respect to their optimization performance. Figure 4 shows the convergence of DSA and GA for the considered two responses. As Munda and Bhattacharyya (2008) did not perform any single objective optimization of the responses, so the optimal solutions as achieved applying DSA cannot be compared with those of the past researchers. The effects of various EMM process parameters on MRR and ROC are also observed through the developed scatter diagrams and the trends exactly match with those identified by Munda and Bhattacharyya (2008).

\section{Table 5}

Results of single objective optimization for Example 2

\begin{tabular}{ccccccccc}
\hline \multirow{2}{*}{ Method } & Response & Objective & Value & $x_{1}$ & $x_{2}$ & $x_{3}$ & $x_{4}$ & $x_{5}$ \\
\hline \multirow{2}{*}{ DSA } & MRR & Maximize & 1.2911 & 0.5000 & 4.1197 & 30.0000 & 51.5735 & 100.0000 \\
& ROC & Minimize & $2.03 \mathrm{E}-05$ & 0.5000 & 2.8408 & 10.9621 & 35.0000 & 173.3133 \\
\hline \multirow{2}{*}{ GA } & MRR & Maximize & 0.9630 & 0.5000 & 3.3169 & 26.0909 & 55.0000 & 287.1363 \\
& ROC & Minimize & $9.91 \mathrm{E}-04$ & 0.5988 & 2.8443 & 15.4795 & 35.2165 & 100.0000 \\
\hline
\end{tabular}
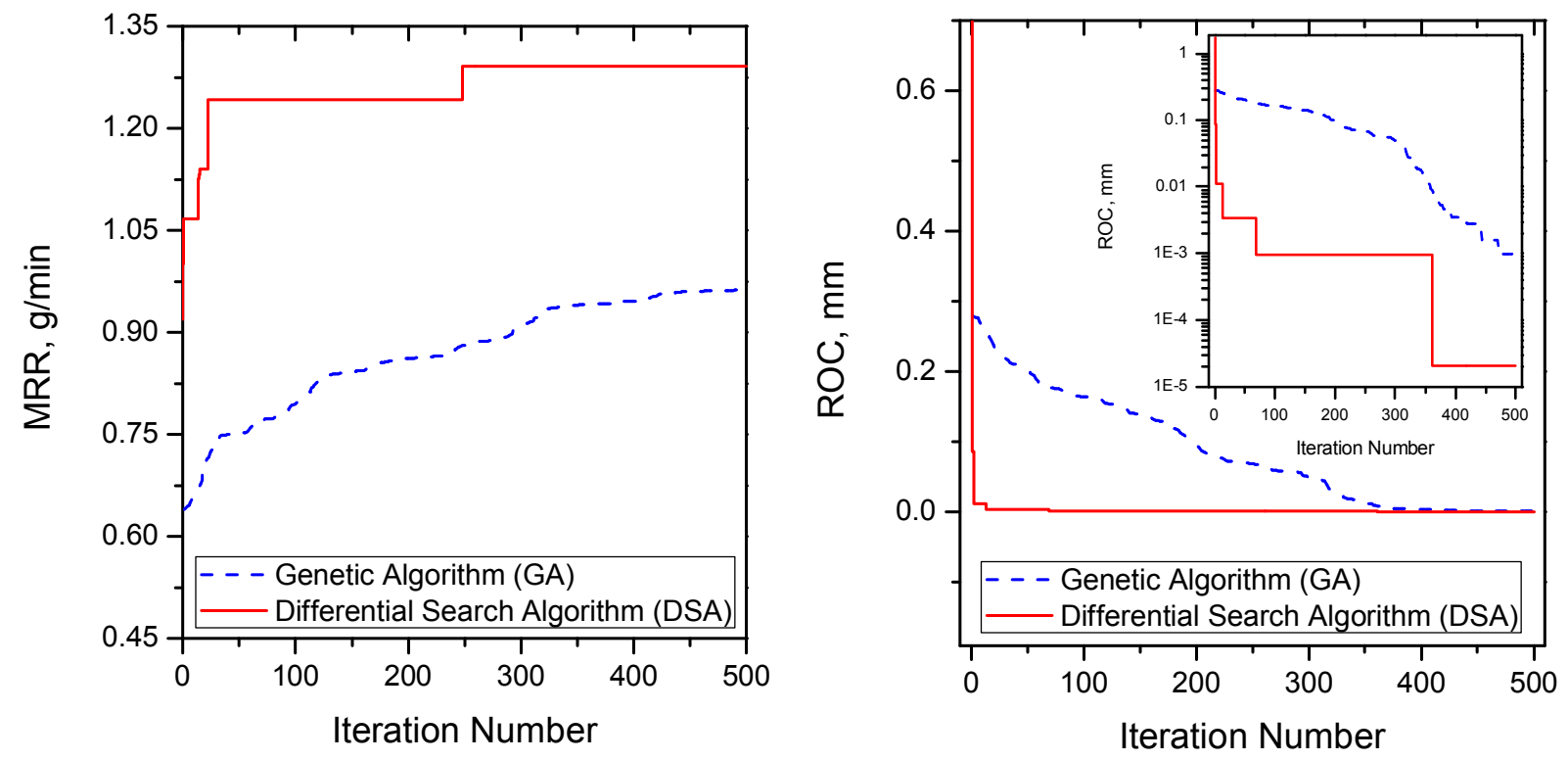

* Inset plot: vertical axis in logarithmic scale

Fig. 4. Convergence diagrams of DSA and GA for Example 2

\section{Multi-objective optimization}

In order to simultaneously optimize both the responses, the following objective function of Eqn. (10) is developed:

Minimize $Z_{2}=\frac{w_{1} \times Y_{R O C}}{(R O C)_{\min }}-\frac{w_{2} \times Y_{M R R}}{(M R R)_{\max }}$,

where $Y_{R O C}$ and $Y_{M R R}$ are the RSM-based equations for ROC and MRR respectively; $w_{1}$ and $w_{2}$ are the weight/priorities assigned to ROC and MRR respectively; and $(R O C)_{\min }$ and $(M R R)_{\max }$ are respectively the optimal values of the considered responses as obtained from the single objective optimization results. Here, equal weights, i.e. $w_{1}=w_{2}=0.50$ are allocated to both the responses. From the results of multi-objective optimization as obtained using DSA and shown in Table 6, it is observed that the maximum MRR value of $0.8354 \mathrm{~g} / \mathrm{min}$ and minimum ROC value of $0.0097 \mathrm{~mm}$ are obtained at the optimal combination of EMM process parameters of pulse on/off ratio $=0.5000$, machining voltage $=$ $4.0974 \mathrm{~V}$, electrolyte concentration $=30.0000 \mathrm{~g} / \mathrm{l}$, voltage frequency $=47.0184 \mathrm{~Hz}$ and tool vibration frequency $=300.0000 \mathrm{~Hz}$. The value of the minimum objective function $\left(Z_{2}\right)$ is found as -0.0810 . 
Munda and Bhattacharyya (2008) determined the maximum MRR value as $0.700 \mathrm{~g} / \mathrm{min}$ and minimum ROC value as around $20 \mu \mathrm{m}$ at the optimal parametric combination of pulse on/off ratio $=1.0$, machining voltage $=3 \mathrm{~V}$, electrolyte concentration $=15 \mathrm{~g} / \mathrm{l}$, voltage frequency $=42.118 \mathrm{~Hz}$ and tool vibration frequency $=300 \mathrm{~Hz}$. Thus, it can be claimed that while using DSA, the value of MRR is increased from $0.700 \mathrm{~g} / \mathrm{min}$ to $0.8354 \mathrm{~g} / \mathrm{min}$ and the ROC value is drastically reduced from $20 \mu \mathrm{m}$ to $9 \mu \mathrm{m}$.

Table 6

Results of multi-objective optimization for Example 2

\begin{tabular}{ccccccc}
\hline$x_{1}$ & $x_{2}$ & $x_{3}$ & $x_{4}$ & $x_{5}$ & ROC & MRR \\
\hline 0.5000 & 4.0974 & 30.0000 & 47.0184 & 300.0000 & 0.0097 & 0.8354 \\
\hline
\end{tabular}

\subsection{Example 3}

After taking into consideration pulse on/off ratio, machining voltage, electrolyte concentration, voltage pulse frequency and tool vibration frequency as the five major EMM process parameters, Munda et al. (2007) studied their influences on micro-spark and stray-current affected zone (MSAZ) (in mm) of the machined surface. The value of MSAZ is quantified as the average difference between the hole radius to the distances between centre of the hole and different points along the curve that indicate the straycurrent and micro-spark affected zone. Each of the EMM process parameters was set at five different levels, as given in Table 7. Based on a central composite half fraction second-order rotatable design plan and using the experimental data, the RSM-based regression equation, as given in Eqn. (11), was developed to study the interactive and higher-order effects of various EMM process parameters on MSAZ.

Table7

EMM process parameters and their levels (Munda et al., 2007)

\begin{tabular}{lcccccc}
\hline Parameter & Unit & \multicolumn{3}{c}{ Levels } & \multicolumn{3}{c}{+1} & +2 \\
& & -2 & -1 & 0 & 2.0 & 2.5 \\
\hline Pulse on/off ratio $\left(x_{1}\right)$ & & 0.5 & 1.0 & 1.5 & 4.0 & 4.5 \\
Machining voltage $\left(x_{2}\right)$ & $\mathrm{V}$ & 2.5 & 3.0 & 3.5 & 25 & 30 \\
Electrolyte concentration $\left(x_{3}\right)$ & $\mathrm{g} / 1$ & 10 & 15 & 20 & 25 \\
Voltage frequency $\left(x_{4}\right)$ & $\mathrm{Hz}$ & 35 & 40 & 45 & 50 & 55 \\
Tool vibration frequency $\left(x_{5}\right)$ & $\mathrm{Hz}$ & 100 & 150 & 200 & 250 & 300 \\
\hline
\end{tabular}

$Y_{M S A Z}=-0.418161-0.468499 x_{1}-0.0470149 x_{2}+0.122239 x_{3}-0.0330168 x_{4}+0.0121153 x_{5}-$

$0.0685619 x_{1}^{2}-0.0267494 x_{2}^{2}-0.00218687 x_{3}^{2}+0.000838756 x_{4}^{2}-4.86869 \times 10^{-6} x_{5}^{2}+0.0829706 x_{1} x_{2}+$

$0.01128426 x_{1} x_{3}+0.00587294 x_{1} x_{4}-0.000567606 x_{1} x_{5}+0.0165958 x_{2} x_{3}$

$-0.000465437 x_{2} x_{4}-0.00117664 x_{2} x_{5}-0.00202633 x_{3} x_{4}-7.65231 \times 10^{-5} x_{3} x_{5}-9.07856 \times 10^{-5} x_{4} x_{5}$

The RSM-based equation depicting the interrelationship between various EMM process parameters and MSAZ is now minimized with respect to the constraints set as $0.5 \leq x_{1} \leq 2.5,2.5 \leq x_{2} \leq 4.5,10 \leq x_{3} \leq$ $30,35 \leq x_{4} \leq 55$ and $100 \leq x_{5} \leq 300$. The results of single objective optimization obtained using DSA and GA are exhibited in Table 8. Employing desirability function approach, Munda et al. (2007) observed the optimal setting of various process parameters as pulse on/off ratio $=2.1618$, machining voltage $=2.8347 \mathrm{~V}$, electrolyte concentration $=10 \mathrm{~g} / \mathrm{l}$, voltage frequency $=35 \mathrm{~Hz}$ and tool vibration frequency $=100 \mathrm{~Hz}$, and a minimum MSAZ value of $1 \times 10^{-4} \mathrm{~mm}$. From Table 8 , it is found that the minimum values of MSAZ are $4.1 \times 10^{-7} \mathrm{~mm}$ and $1.23 \times 10^{-4} \mathrm{~mm}$ respectively when the same optimization problem is solved using DSA and GA techniques. Hence, it can be concluded that the adopted DSA is far superior to GA and desirability function approach with respect to their optimization performance and computational speed. Figure 5 compares the convergence of DSA and GA for this single objective optimization problem. 
Table 8

Results of single objective optimization for Example 3

\begin{tabular}{ccccccccc}
\hline Method & Response & Objective & Value & $x_{1}$ & $x_{2}$ & $x_{3}$ & $x_{4}$ & $x_{5}$ \\
\hline DSA & MSAZ & Minimize & $4.1 \mathrm{E}-07$ & 1.0750 & 4.5000 & 27.8550 & 45.8650 & 161.4100 \\
GA & MSAZ & Minimize & $1.23 \mathrm{E}-04$ & 0.9758 & 3.8396 & 12.3990 & 43.4800 & 129.4400 \\
\hline
\end{tabular}

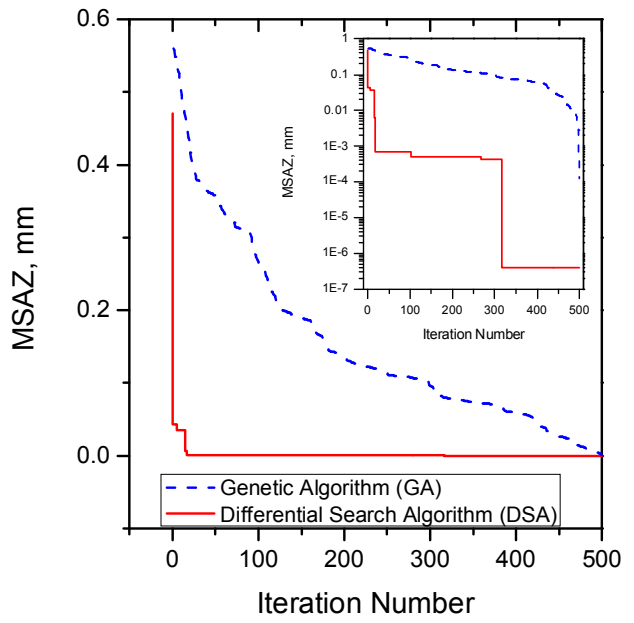

${ }^{*}$ Inset plot: vertical axis in logarithmic scale

Fig. 5. Convergence diagrams of DSA and GA for Example 3

In this example, as there is only a single response, i.e. MSAZ, multi-objective optimization cannot be performed.

\section{Conclusions}

For having enhanced MRR, better surface finish and surface integrity, it is always demanded that the machining parameters of the EMM processes are set at their optimal values. In this paper, three examples leading to parametric optimization of EMM processes are considered and subsequently solved using DSA technique, which is an almost unexplored optimization tool for dealing with complex multi-modal mathematical functions. It is observed that in all the three cases, DSA provides better values of the considered responses (both for single and multi-objective optimization) when compared to those observed by the past researchers. When the optimization performance of DSA is compared with that of GA, the most popular population-based optimization method, it is again found that DSA outperforms GA in all the three examples. Not entirely depending upon the manufactures' data or handbook data, the process engineers can now set the EMM processes at their optimal settings for having improved machining performance. As a future scope, the optimization performance of DSA can also be compared with that of other familiar evolutionary algorithms, like ACO, PSO, ABC, simulated annealing and biogeography-based optimization techniques. The applicability of DSA for parametric optimization of other non-traditional machining processes, like laser machining process, wire electric discharge machining process, plasma arc machining process, electro-chemical discharge machining process can also be experimented.

\section{References}

Bhattacharyya, B., Boloi, B., \& Sridhar, P.S. (2001). Electrochemical micro-machining: new possibilities for micro-manufacturing. Journal of Materials Processing Technology, 113(1-3), 301305.

Bhattacharyya, B., Mitra, S., \& Boro, A.K. (2002). Electrochemical machining: new possibilities for micromachining. Robotics and Computer Integrated Manufacturing, 18(3-4), 283-289. 
Bhattacharyya, B., \& Munda, J. (2003 $\left.{ }^{\mathrm{a}}\right)$. Experimental investigation on the influence of electrochemical machining parameters on machining rate and accuracy in micromachining domain. International Journal of Machine Tools \& Manufacture, 43(13), 1301-1310.

Bhattacharyya, B., \& Munda, J. $\left(2003^{\mathrm{b}}\right)$. Experimental investigation into electrochemical micromachining (EMM) process. Journal of Materials Processing Technology, 140(1-3), 287-291.

Bhattacharyya, B., Munda, J., \& Malapati, M. (2004). Advancement in electrochemical micromachining. International Journal of Machine Tools \& Manufacture, 44(15), 1577-1589.

Bhattacharyya, B., Malapati, M., \& Munda, J. (2005). Experimental study on electrochemical micromachining. Journal of Materials Processing Technology, 169(3), 485-492.

Bhattacharyya, B., Malapati, M., Munda, J., \& Sarkar, A. (2007). Influence of tool vibration on machining performance in electrochemical micro-machining of copper. International Journal of Machine Tools \& Manufacture, 47(2), 335-342.

Cao, G., Dai, Y., \& Yao, L. (2012). Study on mechanism of electrochemical micro-machining of titanium alloys. Applied Mechanics and Materials, 130-134, 2269-2272.

Civicioglu, P. (2012). Transforming geocentric Cartesian coordinates to geodetic coordinates by using differential search algorithm. Computers \& Geosciences, 46, 229-247.

Krebs, J.R. \& Davies, N.B. (1997). Behavioural Ecology: An Evolutionary Approach, Cambridge, UK: Blackwell Publishing.

Kurita, T., Chikamori, K., Kubota, S., \& Hattori, M. (2006). A study of three-dimensional shape machining with an EC $\mu \mathrm{M}$ system. International Journal of Machine Tools \& Manufacture, 46(1213), 1311-1318.

Li, Z., \& Niu, Z. (2010). Process parameter optimization and experimental study of micro-holes in electrochemical micromachining using pulse current. Advanced Materials Research, 135, 293-297.

Malapati, M., \& Bhattacharyya, B. (2011). Investigation into electrochemical micromachining process during micro-channel generation. Materials and Manufacturing Processes, 26(8), 1019-1027.

Malapati, M., Sarkar, A., \& Bhattacharyya, B. (2011). Frequency pulse period and duty factor effects on electrochemical micromachining (EMM). Advanced Materials Research, 264-265, 1334-1339.

Malapati, M., Sarkar, A., \& Bhattacharyya, B. (2012). Micro-channel generation on copper-foil by EMM process. International Journal of Machining and Machinability of Materials, 11(4), 385-404.

Mithu, M.A.H., Fantoni, G., \& Ciampi, J. (2012). Fabrication of micro nozzles and micro pockets by electrochemical micromachining. International Journal of Machining and Machinability of Materials, 11(4), 353-370.

Munda, J., Malapati, M., \& Bhattacharyya, B. (2007). Control of micro-spark and stray-current effect during EMM process. Journal of Materials Processing Technology, 194(1-3), 151-158.

Munda, J., \& Bhattacharyya, B. (2008). Investigation into electrochemical micromachining (EMM) through response surface methodology based approach. International Journal of Advanced Manufacturing Technology, 35(7-8), 821-832.

Munda, J., Malapati, M., \& Bhattacharyya, B. (2010). Investigation into the influence of electrochemical micromachining (EMM) parameters on radial overcut through RSM-based approach. International Journal of Manufacturing Technology and Management, 21(1/2), 54-66.

Rao, R.V., \& Patel, V. (2012). An elitist teaching-learning-based optimization algorithm for solving complex constrained optimization problems. International Journal of Industrial Engineering Computations, 3(4), 535-560.

Samanta, S. \& Chakraborty, S. (2011). Parametric optimization of some non-traditional machining processes using artificial bee colony algorithm. Engineering Applications of Artificial Intelligence, 24, 946-957.

Thanigaivelan, R., \& Arunachalam, R. (2013). Optimization of process parameters on machining rate and overcut in electrochemical micromachining using grey relational analysis. Journal of Scientific and Industrial Research, 72(1), 36-42.

Trianni, V., Tuci, E., Passino, K. \& Marshall, J.R. (2011). Swarm Cognition: an interdisciplinary approach to the study of self-organising biological collectives. Swarm Intelligence, 5, 3-18. 